\title{
Algorithms for Trigonometric Polynomials
}

\author{
Jamie Mulholland \\ Department of Mathematics \\ University of British Columbia \\ Vancouver, B.C. Canada, V6T 1 Z2 \\ jmulholl@cecm.sfu.ca
}

\author{
Michael Monagan \\ Department of Mathematics \\ Simon Fraser University \\ Burnaby, B.C. Canada, V5A 1 S6 \\ monagan@cecm.sfu.ca
}

\begin{abstract}
In this paper we present algorithms for simplifying ratios of trigonometric polynomials and algorithms for dividing, factoring and computing greatest common divisors of trigonometric polynomials, that is, polynomials in $\sin (x)$ and $\cos (x)$.
\end{abstract}

\section{INTRODUCTION}

Let $s$ and $c$ denote $\sin (x)$ and $\cos (x)$ respectively. We are interested in methods for simplifying ratios of trigonometric polynomials, that is, elements of the ring $\mathbb{Q}[s, c] /\left\langle s^{2}+c^{2}-1\right\rangle$. For example, we would like to make the simplification

$$
\frac{(1+c) s+1-c^{2}}{s-2 c^{2}+c^{4}+1} \rightarrow \frac{1+s+c}{\left(1-c^{2}\right) s+1} .
$$

Such simplification problems occur naturally in engineering applications. They will also occur in any computation with trigonometric polynomials that assumes a field, for example, if Gaussian elimination were applied to a matrix of trigonometric polynomials.

One approach to this problem is to try to cancel out a greatest common divisor (GCD) from the numerator and denominator trigonometric polynomials. In the above example the simplified result was obtained by cancelling out the common divisor $s$. What makes this problem non-trivial is that the quotient ring $\mathbb{Q}[s, c] /\left\langle s^{2}+c^{2}-1\right\rangle$ is not a unique factorization domain, thus GCDs are not unique in general. In our example the numerator trigonometric polynomial has the following two irreducible factorizations

$$
(1+c) s+1-c^{2}=s(1+s+c)=(1+c)(1+s-c)
$$

and the denominator polynomial the following two

$$
\begin{aligned}
\left(1+s-2 c^{2}+c^{4}\right) & =s(1+s)\left(2-s-c^{2}\right) \\
& =\frac{1}{2}(1+s+c)(1+s-c)\left(2-s-c^{2}\right) .
\end{aligned}
$$

*This work was supported by NSERC of Canada and the MITACS NCE of Canada.

Permission to make digital or hard copies of all or part of this work for personal or classroom use is granted without fee provided that copies are not made or distributed for profit or commercial advantage, and that copies bear this notice and the full citation on the first page. To copy otherwise, to republish, to post on servers or to redistribute to lists, requires prior specific permission and/or a fee.

ISSAC 2001, UWO, Canada

(C) 2001 ACM 1-58113-218-2/00/0008

$\$ 5.00$
Thus we could also have cancelled out the common factor $1+s+c$ or the common factor $1+s-c$.

The non-uniqueness of factorization in $\mathbb{Q}[s, c] /\left\langle s^{2}+c^{2}-1\right\rangle$ is pointed out by Trotter in [4] though no algorithms are given. In [3], Roach studies this problem in the context of indefinite integration of trigonometric functions. Roach's approach applies to a subset of trigonometric polynomials, those which he terms "co-ordinated". We give some details of his method in section 4 and an implementation of it in REDUCE for comparison with our approach.

In this paper we will show how to compute greatest common divisors of trigonometric polynomials in all cases. To do this we must first understand factorization in the ring $\mathbb{Q}[s, c] /\left\langle s^{2}+c^{2}-1\right\rangle$. We study this in section 2 . We show that $\mathbb{Q}[s, c] /\left\langle s^{2}+c^{2}-1\right\rangle$ is an integral domain and how to do division in it. This facilitates algorithms like fraction-free Gaussian elimination. Next we characterize irreducibles in $\mathbb{Q}[s, c] /\left\langle s^{2}+c^{2}-1\right\rangle$ and show how to determine all distinct factorizations of an element of this ring. An application for factoring trigonometric polynomials would be solving equations involving trigonometric polynomials.

In section 3 we study how to simplify ratios of trigonometric polynomials, i.e. elements of the fraction field of $\mathbb{Q}[s, c] /\left\langle s^{2}+\right.$ $\left.c^{2}-1\right\rangle$. We define and show how to compute greatest common divisors in $\mathbb{Q}[s, c] /\left\langle s^{2}+c^{2}-1\right\rangle$ which gives one solution to the simplification problem, but, surprisingly, this does not always lead to the simplest result. We give a more direct approach which is easy to compute and always yields the simplest result. The ability to simplify ratios of trigonometric polynomials to lowest terms means that algorithms which work over a field can be applied to trigonometric polynomials without "blowing up".

\section{DIVISION AND FACTORIZATION}

To study trigonometric polynomials we consider the ring of polynomials $\mathbb{Q}[s, c]$ modulo $s^{2}+c^{2}-1=0$.

Definition 1. Define the relation $\equiv$ on $\mathbb{Q}[s, c]$ by $p \equiv$ $q \Leftrightarrow s^{2}+c^{2}-1 \mid p-q$.

It is well known that $\equiv$ is an equivalence relation on $\mathbb{Q}[s, c]$. For each $p \in \mathbb{Q}[s, c]$ the equivalence class $[p]=\{q \in \mathbb{Q}[s, c]$ $: p \equiv q\}$. When we speak of a trigonometric polynomial $q$ we mean a representative of the equivalence class $[q]$ in $\mathbb{Q}[s, c] /\left\langle s^{2}+c^{2}-1\right\rangle$. 
Definition 2. Define $\phi: \mathbb{Q}[s, c] \longrightarrow \mathbb{Q}[s, c]$ by $\phi(p)=p \bmod s^{2}+c^{2}-1$ where $s^{2}$ is replaced by $1-c^{2}$.

Thus $\phi(p)$ is an element of the equivalence class $[p]$ which is at most linear in $s$. The following lemma shows that it is unique.

Lemma 1. Let $p \in \mathbb{Q}[s, c]$. Then $\phi(p)$ is the unique element in $\mathbb{Q}[s, c]$ of the form $A(c) s+B(c)$ equivalent to $p$.

Proof: Clearly $\phi(p)$ is linear in $s$ by definition and $\phi(p) \equiv p$. Let $q=A(c) s+B(c)$ such that $q \equiv p$. Write $\phi(p)=a(c) s+$ $b(c)$. Then $\phi(p) \equiv q$ and so $s^{2}+c^{2}-1 \mid(A-a) s+(B-b)$. This is impossible in $\mathbb{Q}[s, c]$ unless $A=a$ and $B=b$.

Since $\left\langle s^{2}+c^{2}-1\right\rangle$ is prime in $\mathbb{Q}[s, c]$ it follows that $\mathbb{Q}[s, c] /\left\langle s^{2}+\right.$ $\left.c^{2}-1\right\rangle$ is an integral domain. We now discuss the degree of a trigonometric polynomial and an analogue to the degreesum formula for polynomials.

Definition 3 (TRIgOnOMETRIC POlynomial DEgREe). For $p \in \mathbb{Q}[s, c] /\left\langle s^{2}+c^{2}-1\right\rangle$ we define the trigonometric degree of $p$ to be $\operatorname{TD}(p)=\operatorname{deg}_{s, c}(\phi(p))$ where $\operatorname{deg}_{s, c}$ means total degree.

Example 1: The polynomial $p=s^{2}+c^{2}$ has total degree 2 in $\mathbb{Q}[s, c]$ but considered as a trigonometric polynomial it has trigonometric degree 0 since $\phi(p)=1$.

LEmma 2 (TRigonometric Degree-Sum Formula). Let $p, q \in \mathbb{Q}[s, c] /\left\langle s^{2}+c^{2}-1\right\rangle$. Then $\operatorname{TD}(p q)=\mathrm{TD}(p)+\mathrm{TD}(q)$.

Proof: Write $\phi(p)=A(c) s+B(c)$ and $\phi(q)=a(c) s+b(c)$. Let $n_{1}=\operatorname{deg}_{c}(A)+1, m_{1}=\operatorname{deg}_{c}(B), n_{2}=\operatorname{deg}_{c}(a)+1$, and $m_{2}=\operatorname{deg}_{c}(b)$. Now

$$
\phi(\phi(p) \phi(q))=(A b+a B) s+\left(B b+A a-A a c^{2}\right) .
$$

We claim that

$$
\operatorname{deg}(\phi(\phi(p) \phi(q)))=\operatorname{deg}(\phi(p))+\operatorname{deg}(\phi(q)) .
$$

This is easy to verify except for the case when $m_{1}=n_{1}, m_{2}=$ $n_{2}$. To determine $\operatorname{deg}(\phi(\phi(p) \phi(q)))$ in this case we argue as follows. Let $A(c)=\sum_{i=0}^{n_{1}-1} A_{i} c^{i}, B(c)=\sum_{i=0}^{m_{1}} B_{i} c^{i}$, $a(c)=\sum_{i=0}^{n_{2}-1} a_{i} c^{i}$, and $b(c)=\sum_{i=0}^{m_{2}} b_{i} c^{i}$. The leading coefficient in $A b+a B$ is $A_{n_{1}-1} b_{m_{2}}+a_{n_{2}-1} B_{m_{1}}$, and the leading coefficient of $B b+A a-A a c^{2}$ is $B_{m_{1}} b_{m_{2}}-A_{n_{1}-1} a_{n_{2}-2}$. If the claim were false then both these coefficients must be zero. The solutions of the system

$$
\left\{A_{n_{1}-1} b_{m_{2}}+a_{n_{2}-1} B_{m_{1}}=0, B_{m_{1}} b_{m_{2}}-A_{n_{1}-1} a_{n_{2}-1}=0\right\}
$$

are the two zero solutions

$$
\left\{A_{n_{1}-1}=0, B_{m_{1}}=0\right\},\left\{a_{n_{2}-1}=0, b_{m_{1}}=0\right\}
$$

and the two complex solutions

$$
\left\{a_{n_{2}-1}= \pm i b_{m_{2}}, A_{n_{1}-1}=\mp i B_{m_{1}}\right\} .
$$

The former contradict the degree of $p$ and $q$ respectively and the latter are not solutions in $\mathbb{Q}$. Therefore $\operatorname{deg}(\phi(\phi(p) \phi(q)))=$ $n_{1}+m_{2}$ and the claim is true for this case.
Note that $p \equiv \phi(p), q \equiv \phi(q), p q \equiv \phi(p q)$ implies $\phi(p) \phi(q) \equiv$ $p q \equiv \phi(p q)$. Therefore $\operatorname{TD}(\phi(p) \phi(q))=\operatorname{deg}(\phi(p q))$. Therefore

$$
\begin{aligned}
\operatorname{TD}(p q) & =\operatorname{deg}(\phi(p q)) \\
& =\operatorname{TD}(\phi(p) \phi(q)) \\
& =\operatorname{deg}(\phi(\phi(p) \phi(q))) \\
& =\operatorname{deg}(\phi(p))+\operatorname{deg}(\phi(q)) \\
& =\operatorname{TD}(p)+\operatorname{TD}(q)
\end{aligned}
$$

\subsection{Tan-half angle substitution}

To simplify ratios of trigonometric polynomials, and to study factorization in the ring $\mathbb{Q}[s, c] /\left\langle s^{2}+c^{2}-1\right\rangle$ we will use the tan-half angle substitution

$$
\sin (x)=\frac{2 \tan (x / 2)}{1+\tan (x / 2)^{2}}, \quad \cos (x)=\frac{1-\tan (x / 2)^{2}}{1+\tan (x / 2)^{2}} .
$$

For the rest of this paper we will denote $\tan (x / 2)$ by $t$. This substitution converts trigonometric polynomials into polynomials in $\mathbb{Q}[t]$ divided by powers of $\left(1+t^{2}\right)$. We employ the tan half-angle substitution instead of the complex exponential mapping simply because it avoids computing in $\mathbb{Q}(i)$ which is more expensive than computing over $\mathbb{Q}$, but otherwise the complex exponential mapping could be used.

\section{Definition 4 (т-Substitution). Define}

$$
\psi_{t}: \mathbb{Q}[s, c] \rightarrow \mathbb{Q}(t) \text { by } \psi_{t}(p(s, c))=p\left(\frac{2 t}{1+t^{2}}, \frac{1-t^{2}}{1+t^{2}}\right) .
$$

We recall the following well known results.

Lemma 3. $\psi_{t}: \mathbb{Q}[s, c] \longrightarrow \mathbb{Q}(t)$ is a ring morphism and the kernel of $\psi_{t}$ is the principle ideal generated by $s^{2}+c^{2}-1$.

Lemma 4. Let $p \in \mathbb{Q}[s, c] /\left\langle s^{2}+c^{2}-1\right\rangle$ with $\operatorname{TD}(p)=d$. Then $\psi_{t}(p)=\frac{a(t)}{\left(1+t^{2}\right)^{d}}$ where $a(t) \in \mathbb{Q}[t]$ such that $\operatorname{deg}_{t}(a) \leq$ $2 d$ and $1+t^{2} \nmid a(t)$.

To make use of this ring morphism we need to be able to invert the t-substitution. We now show how to do this and give some results about the inversion. The inverse t-substitution given by $t=\frac{1-c}{s}$ can be used to convert a rational polynomial in $t$ of the correct form back into a trigonometric polynomial. Two questions arise: (i) What is the correct form? (ii) Does direct substitution of the formula for $t$ always return a trigonometric polynomial? The second question is more involved than it seems. After making the $t=\frac{1-c}{s}$ substitution we have a rational trigonometric polynomial and so to get a trigonometric polynomial we need to do trigonometric polynomial division. This is a problem in its own right. There are simple ways to do the division in this case but we will not consider them since we can answer both questions by cleverly making the inverse $t$-substitution using resultants. How we do this is illustrated in the following theorem and subsequent comments. However, first we must state the following useful lemma.

LEMMA 5. Let $a(t)=\sum_{i=0}^{2 n} v_{i} t^{i}$ such that $v_{2 n}=\sum_{i=1}^{n}(-1)^{i+1} v_{2(n-i)}$ and $v_{2 n-1}=\sum_{i=1}^{n-1}(-1)^{i+1} v_{2(n-i)-1}$ then $1+t^{2} \mid a(t)$. 
Proof: Consider $b(t)=\sum_{i=0}^{2 n-2} b_{i} t^{i}$ where $b_{i}=\sum_{k=0}^{i / 2}(-1)^{k} v_{i-2 k}$ if $i$ even and $b_{i}=\sum_{k=0}^{(i-1) / 2}(-1)^{k} v_{i-2 k}$ if $i$ odd. We leave it to the reader to verify $a(t)=\left(1+t^{2}\right) \cdot b(t)$.

Lemma 6 . Let $n \in \mathbb{N}$. For each polynomial $a(t)$ with $1+t^{2} \nmid a(t)$ and $\operatorname{deg}_{t}(a) \leq 2 n$, the resultant

$$
\operatorname{res}_{t}\left(a(t)-X\left(1+t^{2}\right)^{n}, s t+(c-1)\right)=p(s, c) X+q(s, c)
$$

where $p(s, c), q(s, c) \in \mathbb{Q}[s, c]$. Moreover, $\phi(p)=-2^{n}(1-c)^{n}$ and $\phi(p) \mid \phi(q)$ in $\mathbb{Q}[s, c]$ and $\operatorname{TD}(q(s, c))=2 n$.

Proof: Write $a(t)=\sum_{i=0}^{2 n} v_{i} t^{i}$, where of course if $\operatorname{deg}_{t}(a)<$ $2 n$ then $v_{i}=0$ for $i>\operatorname{deg}_{t}(a(t))$. The resultant in the lemma is the determinant of Sylvesters' matrix

$$
S=\left[\begin{array}{ccccc}
v_{2 n}-\left(\begin{array}{c}
n \\
0
\end{array}\right) X & v_{2 n-1} & v_{2 n-2}-\left(\begin{array}{c}
n \\
1
\end{array}\right) X & v_{2 n-3} & \ldots \\
s & c-1 & 0 & 0 & \ldots \\
0 & s & c-1 & 0 & \ldots \\
0 & 0 & s & c-1 & \ldots \\
\vdots & \vdots & \vdots & \vdots & \vdots
\end{array}\right]
$$

The reader can verify that $\operatorname{det}(S)=p(s, c) X+q(s, c)$ where $\phi(p(s, c))=-2^{n}(1-c)^{n}$ and $\phi(q(s, c))=(1-c)^{n}\left[\sum_{i=0}^{n} v_{2(n-i)}\right.$ $\left.(1+c)^{i}(1-c)^{n-i}+\sum_{i=0}^{n-1} v_{2(n-i)-1} s(1+c)^{i}(1-c)^{n-i-1}\right]$. The only thing left to show is that $\operatorname{TD}(q(s, c))=2 n$, that is, $\operatorname{deg}_{s, c} \phi(q(s, c))=2 n$. If the total degree of $\phi(q(s, c))$ is $<2 n$ then both the coefficient of $c^{n}$ and $s c^{n}$ must be zero in the second factor of $\phi(q(s, c))$. This means that $v_{2 n}=$ $\sum_{i=1}^{n}(-1)^{i+1} v_{2(n-i)}$ and $v_{2 n-1}=\sum_{i=1}^{n-1}(-1)^{i+1} v_{2(n-i)-1}$, and by Lemma $51+t^{2}$ divides $a(t)$. This is a contradiction. Therefore $\operatorname{TD}(q(s, c))=2 n$.

TheOREM 1. Let $n \in \mathbb{N}$. Let $a(t)$ be a polynomial such that $1+t^{2} \nmid a(t)$ and $\operatorname{deg}_{t}(a) \leq 2 n$. Then there exists a unique trigonometric polynomial $\hat{a}$ of trigonometric degree $n$ such that $\psi_{t}(\hat{a})=\frac{a(t)}{\left(1+t^{2}\right)^{n}}$.

Proof: From the previous lemma we have that

$\operatorname{res}_{t}\left(a(t)-X\left(1+t^{2}\right)^{n}, s t+(c-1)\right)=p(s, c) X+q(s, c)$

where $\phi(p) \mid \phi(q)$ in $\mathbb{Q}[s, c], \operatorname{TD}(p)=n$ and $\operatorname{TD}(q)=2 n$. Solving $p(s, c) X+q(s, c)=0$ for $X$ in $\mathbb{Q}[s, c] /\left\langle s^{2}+c^{2}-1\right\rangle$ we get

$$
X=\frac{\phi(q)}{\phi(p)} \in \mathbb{Q}[s, c] /\left\langle s^{2}+c^{2}-1\right\rangle .
$$

Now $X \in \mathbb{Q}[s, c] /\left\langle s^{2}+c^{2}-1\right\rangle$ has trigonometric degree $n$ and from the definition of the resultant we have

$$
\psi_{t}(X)=\frac{a(t)}{\left(1+t^{2}\right)^{n}} .
$$

To show uniqueness, let $\hat{a}, \hat{b} \in \mathbb{Q}[s, c] /\left\langle s^{2}+c^{2}-1\right\rangle$ such that $\psi_{t}(\hat{a})=\psi_{t}(\hat{b})=\frac{a(t)}{\left(1+t^{2}\right)^{n}}$. Since $\psi_{t}$ is a ring morphism then $\psi_{t}(\hat{a}-\hat{b})=0$ so $\hat{a}-\hat{b} \in \operatorname{ker} \psi_{t}=\left\langle s^{2}+c^{2}-1\right\rangle$, thus $\hat{a}=\hat{b}$.

Theorem 1 says that every rational polynomial of the form $\frac{a(t)}{\left(1+t^{2}\right)^{n}}$ where $a \in \mathbb{Q}[t], 1+t^{2} \nmid a(t)$ and $\operatorname{deg}_{t}(a) \leq 2 n$ is the image under $\psi_{t}$ of a trigonometric polynomial of degree $n$. In what follows we denote $\psi_{t}^{-1}\left(\frac{a(t)}{\left(1+t^{2}\right)^{n}}\right)=X(s, c)$ where $X(s, c)$ is the unique polynomial in the proof.

\subsection{Division in $\mathbb{Q}[s, c] /\left\langle s^{2}+c^{2}-1\right\rangle$}

The following theorem shows when one trigonometric polynomial divides another in $\mathbb{Q}[s, c] /\left\langle s^{2}+c^{2}-1\right\rangle$ and the proof shows how to compute the quotient. In what follows we denote by NU the numerator of a rational function, for example, $\mathrm{NU}\left(\frac{a(t)}{\left(1+t^{2}\right)^{n}}\right)=a(t)$.

Theorem 2. For $a, b \in \mathbb{Q}[s, c] /\left\langle s^{2}+c^{2}-1\right\rangle$

$$
\begin{array}{ccl}
a \mid b \Leftrightarrow \quad(i) & \operatorname{TD}(a) \leq \mathrm{TD}(b) \\
& (\text { ii }) \quad \mathrm{NU}\left(\psi_{t}(a)\right) \mid \mathrm{NU}\left(\psi_{t}(b)\right) \quad \text { in } \mathbb{Q}[\mathrm{t}] \\
& (\text { iii }) & \operatorname{deg}\left(\mathrm{NU}\left(\psi_{t}(b)\right)\right)-\operatorname{deg}\left(\mathrm{NU}\left(\psi_{t}(a)\right)\right) \\
& \leq 2(\mathrm{TD}(b)-\operatorname{TD}(a))
\end{array}
$$

Proof: $(\Rightarrow)$ Let $q$ be such that $b=q a$ in $\mathbb{Q}[s, c] /\left\langle s^{2}+c^{2}-1\right\rangle$. Then $\exists r \in \mathbb{Q}[s, c]$ such that $b=q a+r\left(s^{2}+c^{2}-1\right)$ in $\mathbb{Q}[s, c]$. By the trigonometric degree-sum formula, Lemma $2, \mathrm{TD}(b)=\mathrm{TD}(q)+\mathrm{TD}(a)$. Moreover,

$$
\begin{aligned}
\psi_{t}(b) & =\psi_{t}\left(q a+r\left(s^{2}+c^{2}-1\right)\right) \\
& =\psi_{t}(q) \psi_{t}(a)+\psi_{t}(r) \cdot 0 \\
& =\psi_{t}(q) \psi_{t}(a) .
\end{aligned}
$$

Expanding this equation we have

$$
\frac{\mathrm{NU}\left(\psi_{t}(b)\right)}{\left(1+t^{2}\right)^{d_{b}}}=\frac{\mathrm{NU}\left(\psi_{t}(q)\right)}{\left(1+t^{2}\right)^{d_{q}}} \cdot \frac{\mathrm{NU}\left(\psi_{t}(a)\right)}{\left(1+t^{2}\right)^{d_{a}}}
$$

where $d_{x}=\operatorname{TD}(x)$ for $x=a, b, q$. Since $d_{b}=d_{q}+d_{a}$ then $\mathrm{NU}\left(\psi_{t}(b)\right)=\mathrm{NU}\left(\psi_{t}(q)\right) \cdot \mathrm{NU}\left(\psi_{t}(a)\right)$. Moreover,

$$
\begin{aligned}
\operatorname{deg}(\mathrm{NU} & \left.\left(\psi_{t}(b)\right)\right)-\operatorname{deg}\left(\mathrm{NU}\left(\psi_{t}(a)\right)\right) \\
& =\operatorname{deg}\left(\mathrm{NU}\left(\psi_{t}(q)\right)\right) \\
& \leq 2 \mathrm{TD}(q) \\
& =2(\operatorname{TD}(b)-\operatorname{TD}(a)) .
\end{aligned}
$$

This proves $(\Rightarrow)$. For $(\Leftarrow)$ suppose that the three conditions hold as stated in the theorem. Then $\mathrm{NU}\left(\psi_{t}(b)\right)=q(t)$. $\mathrm{NU}\left(\psi_{t}(a)\right)$ for some $q \in \mathbb{Q}[t]$. So

$$
\frac{\mathrm{NU}\left(\psi_{t}(b)\right)}{\left(1+t^{2}\right)^{d_{b}}}=\frac{q(t)}{\left(1+t^{2}\right)^{d_{b}-d_{a}}} \cdot \frac{\mathrm{NU}\left(\psi_{t}(a)\right)}{\left(1+t^{2}\right)^{d_{a}}} .
$$

Since

$$
\begin{aligned}
\operatorname{deg}_{t}(q) & =\operatorname{deg}\left(\mathrm{NU}\left(\psi_{t}(b)\right)-\operatorname{deg}\left(\mathrm{NU}\left(\psi_{t}(a)\right)\right)\right. \\
& \leq 2(\operatorname{TD}(b)-\operatorname{TD}(a)) \quad \text { from (ii) } \\
& =2\left(d_{b}-d_{a}\right)
\end{aligned}
$$

we are guaranteed (by Theorem 1) that

$$
\widehat{q}=\psi_{t}^{-1}\left(\frac{q(t)}{\left(1+t^{2}\right)^{d_{b}-d_{a}}}\right) \in \mathbb{Q}[s, c] /\left\langle s^{2}+c^{2}-1\right\rangle
$$

Therefore, $a=\widehat{q} \cdot b$ in $\mathbb{Q}[s, c] /\left\langle s^{2}+c^{2}-1\right\rangle$.

Corollary 1. Let $p \in \mathbb{Q}[s, c] /\left\langle s^{2}+c^{2}-1\right\rangle$ such that $\operatorname{TD}(p) \geq$ 1. If $\operatorname{deg}\left(\mathrm{NU}\left(\psi_{t}(p)\right)\right) \leq 2 \mathrm{TD}(p)-2$ then $c+1 \mid p$.

Proof: Since $\psi_{t}(c+1)=\frac{2}{1+t^{2}}$, then from the previous theorem we have

$$
\begin{array}{rll}
c+1 \mid p \Leftrightarrow \quad & (\text { i }) \quad 1 \leq \mathrm{TD}(p) \\
& (\text { ii }) \quad 2 \mid \mathrm{NU}\left(\psi_{t}(p)\right) \\
& (\text { iii }) \quad \operatorname{deg}\left(\mathrm{NU}\left(\psi_{t}(p)\right)\right) \leq 2(\mathrm{TD}(p)-1) .
\end{array}
$$

Since (i),(ii),(iii) are all true then $c+1 \mid p$. 


\subsection{Irreducibility in $\mathbb{Q}[s, c] /\left\langle s^{2}+c^{2}-1\right\rangle$}

In this section we will identify irreducibles in $\mathbb{Q}[s, c] /\left\langle s^{2}+\right.$ $\left.c^{2}-1\right\rangle$. Since the units in $\mathbb{Q}[s, c] /\left\langle s^{2}+c^{2}-1\right\rangle$ are the nonzero rational numbers, this leads to the following definition of the irreducibles in $\mathbb{Q}[s, c] /\left\langle s^{2}+c^{2}-1\right\rangle$.

Definition 5. A non-zero element $p \in \mathbb{Q}[s, c] /\left\langle s^{2}+c^{2}-1\right\rangle$ is irreducible if

(a) $p$ is not in $\mathbb{Q}$ (i.e. $p$ is not a unit)

(b) whenever $p=a b$ either $a$ or $b$ is in $\mathbb{Q}$.

TheOREM 3. Let $p \in \mathbb{Q}[s, c] /\left\langle s^{2}+c^{2}-1\right\rangle$ such that $p$ is not a unit (i.e. $\operatorname{TD}(p) \geq 1$ ),

(a) if $\operatorname{TD}(p)=1$ then $p$ is irreducible.

(b) if $\operatorname{TD}(p)>1$ then $p$ is irreducible $\Leftrightarrow$

(i) $\quad \operatorname{deg}_{t}\left(\mathrm{NU}\left(\psi_{t}(p)\right)\right) \geq 2 \mathrm{TD}(p)-1$,

(ii) $\quad \mathrm{NU}\left(\psi_{t}(p)\right)$ is irreducible in $\mathbb{Q}[t]$ or

$\mathrm{NU}\left(\psi_{t}(p)\right)$ is a product of two irreducible

polynomials in $\mathbb{Q}[t]$ each of odd degree.

Proof: If $\operatorname{TD}(p)=1$ then $p$ is irreducible by the trigonometric degree-sum formula. Now suppose $\operatorname{TD}(p) \geq 2$.

$(\Rightarrow)$ Suppose that (i) or (ii) does not hold. If (i) does not hold then $c+1 \mid p$ by Corollary 1 , so $p$ is reducible. So suppose (i) holds but (ii) does not hold, then we must have $\mathrm{NU}\left(\psi_{t}(p)\right)$ is reducible but is not the product of two irreducible polynomials in $\mathbb{Q}[t]$ of odd degree. Write

$$
\mathrm{NU}\left(\psi_{t}(p)\right)=q_{1}(t) \cdot q_{2}(t),
$$

for some $q_{1}, q_{2} \in \mathbb{Q}[t]$ such that $\operatorname{deg}\left(q_{1}\right), \operatorname{deg}\left(q_{2}\right) \geq 1, q_{1}$ irreducible and if $q_{2}$ irreducible then $\operatorname{deg}\left(q_{1}\right), \operatorname{deg}\left(q_{2}\right)$ cannot both be odd. Since (i) holds we have two case to consider, namely

$$
\begin{aligned}
& \text { (1) } \operatorname{deg}_{t}\left(\mathrm{NU}\left(\psi_{t}(p)\right)\right)=2 d \text { or } \\
& \text { (2) } \operatorname{deg}_{t}\left(\mathrm{NU}\left(\psi_{t}(p)\right)\right)=2 d-1 .
\end{aligned}
$$

Case (1): If degrees of $q_{1}$ and $q_{2}$ are both even then we can split up $\psi_{t}(p)$ as

$$
\psi_{t}(p)=\frac{q_{1}}{\left(1+t^{2}\right)^{d_{1}}} \cdot \frac{q_{2}}{\left(1+t^{2}\right)^{d_{2}}}
$$

where $d_{i}=(1 / 2) \operatorname{deg}_{t}\left(q_{i}\right)$ for $i=1,2$. If degrees are both odd then $q_{2}$ must be reducible. Moreover, $q_{2}$ must have an irreducible factor of odd degree, say $q_{3}$. Write $q_{2}=q_{3} \cdot q_{4}$ (note $\operatorname{deg}_{t}\left(q_{4}\right)$ even). We can split up $\psi_{t}(p)$ as

$$
\psi_{t}(p)=\frac{q_{1} \cdot q_{3}}{\left(1+t^{2}\right)^{d_{1}}} \cdot \frac{q_{4}}{\left(1+t^{2}\right)^{d_{2}}}
$$

where $d_{1}=(1 / 2)\left(\operatorname{deg}_{t}\left(q_{1}\right)+\operatorname{deg}_{t}\left(q_{3}\right)\right)$ and $d_{2}=(1 / 2) \operatorname{deg}_{t}\left(q_{4}\right)$.

Case (2): Exactly one of $q_{1}$ and $q_{2}$ is of odd degree, say $q_{1}$. Split up $\psi_{t}(p)$ as

$$
\psi_{t}(p)=\frac{q_{1}}{\left(1+t^{2}\right)^{d_{1}}} \cdot \frac{q_{2}}{\left(1+t^{2}\right)^{d_{2}}}
$$

where $d_{1}=(1 / 2)\left(\operatorname{deg}_{t}\left(q_{1}\right)+1\right)$ and $d_{2}=(1 / 2)\left(\operatorname{deg}_{t}\left(q_{2}\right)\right)$.

In either case we can split up $\psi_{t}(p)$ as

$$
\psi_{t}(p)=\frac{\hat{q_{1}}}{\left(1+t^{2}\right)^{d_{1}}} \cdot \frac{\hat{q_{2}}}{\left(1+t^{2}\right)^{d_{2}}}
$$

where $2 d_{i} \geq \operatorname{deg}_{t}\left(\hat{q}_{i}\right)$ and $d_{i} \geq 1, i=1,2$. By Theorem 1 both of these correspond to trigonometric polynomials in $\mathbb{Q}[s, c] /\left\langle s^{2}+c^{2}-1\right\rangle$ with degrees $d_{1}$ and $d_{2}$ respectively, where $d_{1}, d_{2} \geq 1$. Thus $p$ is reducible.

$(\Leftarrow)$ Suppose (i) and (ii) hold. Towards a contradiction suppose $p$ is reducible. Write $p=a \cdot b$ where $a, b \in \mathbb{Q}[s, c] /\left\langle s^{2}+\right.$ $\left.c^{2}-1\right\rangle$, and $\operatorname{TD}(a) \geq 1, \operatorname{TD}(b) \geq 1$. Now

$$
\begin{aligned}
\psi_{t}(p) & =\psi_{t}(a) \cdot \psi_{t}(b) \\
& =\frac{\mathrm{NU}\left(\psi_{t}(a)\right)}{\left(1+t^{2}\right)^{d_{a}}} \cdot \frac{\mathrm{NU}\left(\psi_{t}(b)\right)}{\left(1+t^{2}\right)^{d_{b}}}
\end{aligned}
$$

where $d_{a}=\operatorname{TD}(a)$ and $d_{b}=\operatorname{TD}(b)$. By the trig degree-sum formula $d_{a}+d_{b}=\mathrm{TD}(p)$. If $\operatorname{deg}_{t}\left(\mathrm{NU}\left(\psi_{t}(a)\right)\right)=0$ then

$$
\begin{aligned}
\operatorname{deg}_{t}\left(\mathrm{NU}\left(\psi_{t}(p)\right)\right) & =\operatorname{deg}\left(\mathrm{NU}\left(\psi_{t}(b)\right)\right) \\
& \leq 2 d_{b} \\
& =2\left(\operatorname{TD}(p)-d_{a}\right) \\
& =2 \operatorname{TD}(p)-2 d_{a} \\
& \leq 2 \operatorname{TD}(p)-2 \quad \text { since } \mathrm{d}_{\mathrm{a}} \geq 1 \\
& <2 \mathrm{TD}(p)-1 .
\end{aligned}
$$

This contradicts (i). Similarly if $\operatorname{deg}_{t}\left(\mathrm{NU}\left(\psi_{t}(b)\right)\right)=0$. So $\mathrm{NU}\left(\psi_{t}(a)\right), \mathrm{NU}\left(\psi_{t}(b)\right) \notin \mathbb{Q}$. Thus $\mathrm{NU}\left(\psi_{t}(p)\right)$ is reducible in $\mathbb{Q}[t]$. Since (ii) holds and $\mathrm{NU}\left(\psi_{t}(p)\right)$ is reducible then both $\mathrm{NU}\left(\psi_{t}(a)\right), \mathrm{NU}\left(\psi_{t}(b)\right)$ are irreducible and $\operatorname{deg}_{t}(\mathrm{NU}(\psi(a)))$, $\operatorname{deg}_{t}(\mathrm{NU}(\psi(b)))$ are odd. Let $\widehat{d_{a}}=\operatorname{deg}_{t}(\mathrm{NU}(\psi(a)))$ and $\widehat{d_{b}}=$ $\operatorname{deg}_{t}(\mathrm{NU}(\psi(b)))$. Since $\widehat{d_{a}}, \widehat{d_{b}}$ are both odd and $\leq 2 d_{a}$ and $2 d_{b}$ respectively then $\widehat{d_{a}} \leq 2 d_{a}-1$ and $\widehat{d_{b}} \leq 2 d_{b}-1$.

Since (i) holds then

$$
\operatorname{deg}_{t}\left(\mathrm{NU}\left(\psi_{t}(p)\right)\right)=2 d \text { or } 2 d-1 .
$$

If $\operatorname{deg}_{t}(\mathrm{NU}(\psi(p)))=2 d$ then $2 d=\widehat{d_{a}}+\widehat{d_{b}}<2 d_{a}+2 d_{b}=2 d$. If $\operatorname{deg}_{t}(\mathrm{NU}(\psi(p)))=2 d-1$ then $2 d-1=\widehat{d_{a}}+\widehat{d_{b}}<2 d_{a}+$ $2 d_{b}-2=2 d-2$ which implies $2 d \leq 2 d-1$. In each case we get a contradiction. Therefore $p$ is irreducible.

It follows from Theorem 3 that $s, 1+c$ and $1-c$ are all irreducible in $\mathbb{Q}[s, c] /\left\langle s^{2}+c^{2}-1\right\rangle$, hence the trigonometric polynomial $s^{2}=1-c^{2}$ has two distinct factorizations, namely, $s \times s$ and $(1+c)(1-c)$. Notice also that Theorem 3 says that an irreducible trigonometric polynomial may not have an irreducible image under $\psi_{t}$. Moreover, an irreducible image does not imply that the trigonometric polynomial is irreducible either as the numerator of the image of $c+1$ has degree 0 in $t$.

\subsection{Factorization in $\mathbb{Q}[s, c] /\left\langle s^{2}+c^{2}-1\right\rangle$}

In this subsection we will sketch, by way of examples, an algorithm for determining all factorizations of a trigonometric polynomial. We will not present an explicit algorithm for doing this because the details are many and messy. A Maple code for doing this is available from the authors.

Theorem 3 characterizes the irreducible trigonometric polynomials in terms of their images under the $t$-substitution. So to find all factorizations of a trigonometric polynomial we need to factor the numerator and look at combinations of pairs of the odd degree factors, and also take into account the possibility of $(1+c)$ factors. 
Example 2: Let $p=s c$.

First we find the image of $p$ under $\psi_{t}$ :

$$
\psi_{t}(p)=\psi_{t}(s c)=\frac{2 t(1+t)(1-t)}{\left(1+t^{2}\right)^{2}} .
$$

Each factor of $p$, under $\psi_{t}$, must have denominator $1+t^{2}$ to some power, thus $p$ can have at most two factors since the power of $1+t^{2}$ in the denominator of $\psi_{t}(p)$ is 2. Applying Theorem 3 we see that the possible factorizations of $\psi_{t}(p)$ are:

$$
\begin{gathered}
2 \frac{t}{1+t^{2}} \cdot \frac{(1+t)(1-t)}{1+t^{2}}, 2 \frac{1+t}{1+t^{2}} \cdot \frac{t(1-t)}{1+t^{2}}, \text { and } \\
2 \frac{1-t}{1+t^{2}} \cdot \frac{t(1+t)}{1+t^{2}}
\end{gathered}
$$

These correspond to the three distinct factorizations of $p$

$$
\begin{aligned}
& 2(1 / 2 s) \cdot c=s c, \quad 2(1 / 2(c+s+1)) \cdot(1 / 2(c+s-1)) \\
& \quad=1 / 2(c+s+1)(c+s-1), \text { and } \\
& 2(1 / 2(c-s+1)) \cdot(1 / 2(-c+s+1)) \\
& \quad=1 / 2(c-s+1)(s-c+1) .
\end{aligned}
$$

Example 3 (from the introduction): Let $p=(1+c) s+1-c^{2}$. Again, we first find the image of $p$ under $\psi_{t}$ :

$$
\psi_{t}(p)=\frac{4 t(1+t)}{\left(1+t^{2}\right)^{2}} .
$$

Each factor of $p$, under $\psi_{t}$, must have denominator $1+t^{2}$ to some power, thus $p$ can have at most two factors. A possible factorization of $\psi_{t}(p)$ is

$$
4 \frac{t}{1+t^{2}} \cdot \frac{1+t}{1+t^{2}}
$$

However, there is still another factorization

$$
4 \frac{1}{1+t^{2}} \cdot \frac{t(1+t)}{1+t^{2}}
$$

This factorization follows from Corollary 1. We can easily see that there are no other factorizations of $\psi_{t}(p)$ in which each factor corresponds to a trigonometric polynomial. Therefore the two distinct factorizations of $p$ are:

$$
\begin{gathered}
4(1 / 2 s)(1 / 2(c+s+1))=s(c+s+1), \\
4(1 / 2(1+c))(1 / 2(s-c+1))=(1+c)(s-c+1) .
\end{gathered}
$$

We end this section by noting that the number of distinct factorizations of $p \in \mathbb{Q}[s, c] /\left\langle s^{2}+c^{2}-1\right\rangle$ grows exponentially with the number of distinct odd degree factors of $a(t)=$ $\mathrm{NU}\left(\psi_{t}(p)\right)$. For example, if $\operatorname{TD}(p)=d$ and $a(t)$ factors into $2 d$ distinct linear factors then there are $1 \times 3 \times 5 \times \ldots \times(2 d-1)$ distinct factorizations of $p$ and $\left(\begin{array}{c}2 d \\ 2\end{array}\right)=2 d^{2}-d$ distinct irreducible divisors of $p$. Instead of computing all trigonometric factorizations of a trigonometric polynomial $p$, an application may require only a single factorization or the set of irreducible trigonometric polynomial divisors of $p$. This information is also easily determined from the factorization of $a(t)$ and application of Theorem 3 .

\section{GCDS AND SIMPLIFICATION}

In this section we define the trigonometric GCD of two trigonometric polynomials and give an algorithm for computing it. The classical definition of GCD for a commutative ring $R$, as stated, for example, by Hungerford in [2], is

Definition 6 (ClASSiCAL GCD). Let $R$ be a commutative ring and let $a, b \in R$. An element $g \in R$ is a greatest common divisor of $a$ and $b$ if

(i) $g \mid a$ and $g \mid b$, and

(ii) if $p \mid a$ and $p \mid b$ then $p \mid g$.

Example 4 below shows that under this definition a GCD may not exist even when common divisors exist. This is not useful for computational purposes so we are led to consider the following definition for a GCD. The two definitions are equivalent when we are in a UFD.

Definition 7 (ALternative GCD). Let $R$ be a commutative ring and let $a, b \in R$. An element $g \in R$ is a greatest common divisor of $a$ and $b$ if

(i) $g \mid a$ and $g \mid b$, and

(ii) if $p \mid(a / g)$ and $p \mid(b / g)$ then $p$ is a unit.

Example 4: Let $a=s(1+c), b=-c^{2}+c s+s+1$. Using the methods of Section 2.4 we find that $a$ and $b$ have exactly the following factorizations:

$$
\begin{gathered}
a=s(c+1), \\
b=(c+s+1) s=(-c+s+1)(1+c) .
\end{gathered}
$$

Now, $s$ and $1+c$ divide both $a$ and $b$, hence under the classical definition of GCD we would have that the GCD $g$, if it exists, of $a$ and $b$ is divisible by both $s$ and $1+c$. Thus, $s, 1+c \mid g$ and $g \mid a=s(1+c)$ so $g=s(1+c)$ (up to multiplication by a unit). But $g=s(1+c) \nmid b$, a contradiction. However, we have that both $s$ and $1+c$ are GCDs of $a$ and $b$ according to the alternative definition.

The previous example shows that GCDs are not unique. The next example shows that two GCDs may not even have the same trigonometric degree.

Example 5: Let $a=-5 c^{3}+5 s c^{2}-5 c^{2}+2 c s+c+9 s+9$, $b=-c^{5}+17 c^{4}-7 s c^{4}+6 c^{3}-16 s c^{3}+2 c^{2}-14 s c^{2}-21 c-$ $24 c s-3-3 s$. The images of $a$ and $b$ under $\psi_{t}$ are:

$$
\begin{gathered}
\psi_{t}(a)=8 \frac{t(t+1)\left(t^{3}+2\right)(t+2)}{\left(1+t^{2}\right)^{3}}, \\
\psi_{t}(b)=32 \frac{t(t+1)\left(t^{3}+2\right)\left(t^{5}-2\right)}{\left(1+t^{2}\right)^{5}} .
\end{gathered}
$$

We cannot take the image of the GCD to be $t(t+1)\left(t^{3}+2\right)$ for if we did we cannot partition the three $\left(1+t^{2}\right)$ factors in the denominator of $\psi_{t}(a)$ between $t(t+1)\left(t^{3}+2\right)$ and the cofactor image $(t+2)$ so that both are images of a trigonometric polynomials. We can split these images of trigonometric polynomials as follows:

$$
\psi_{t}(a)=8 \frac{t(t+1)}{1+t^{2}} \cdot \frac{\left(t^{3}+2\right)(t+2)}{\left(1+t^{2}\right)^{2}} \text { and }
$$




$$
\psi_{t}(b)=32 \frac{t(t+1)}{1+t^{2}} \cdot \frac{\left(t^{3}+2\right)\left(t^{5}-2\right)}{\left(1+t^{2}\right)^{4}} .
$$

We can also split them as:

$$
\begin{gathered}
\psi_{t}(a)=8 \frac{t\left(t^{3}+2\right)}{\left(1+t^{2}\right)^{2}} \cdot \frac{(t+1)(t+2)}{1+t^{2}} \text { and } \\
\psi_{t}(b)=32 \frac{t\left(t^{3}+2\right)}{\left(1+t^{2}\right)^{2}} \cdot \frac{(t+1)\left(t^{5}-2\right)}{\left(1+t^{2}\right)^{3}} .
\end{gathered}
$$

The first way we have the common factor $\frac{t(t+1)}{1+t^{2}}$ which corresponds to the GCD $s-c+1$ of $a$ and $b$ whereas in the second way we have the common factor $\frac{t\left(t^{3}+2\right)}{\left(1+t^{2}\right)^{2}}$ which corresponds to the GCD $c^{2}+2 c s-2 c+2 s+1$.

This example shows that two GCDs may have different trigonometric degrees. It seems reasonable that a GCD of highest trigonometric degree is the one we really are interested in. This motivates the following definition for a GCD in $\mathbb{Q}[s, c] /\left\langle s^{2}+c^{2}-1\right\rangle$.

DEFINITION 8 (TRIGONOMETRIC GCD). Let $a, b \in$ $\mathbb{Q}[s, c] /\left\langle s^{2}+c^{2}-1\right\rangle$. An element $g \in \mathbb{Q}[s, c] /\left\langle s^{2}+c^{2}-\right.$ 1) is called a trigonometric greatest common divisor (trig $G C D)$ of $a$ and $b$ if

(i) $g \mid a$ and $g \mid b$, and

(ii) if $p \mid(a / g)$ and $p \mid(b / g)$ then $p$ is a unit.

(iii) if $h$ satisfies (i) and (ii) then $\operatorname{TD}(g) \geq \operatorname{TD}(h)$

Following the method in the previous example we now present an algorithm for computing trigonometric greatest common divisors.

\section{Algorithm [trig GCD]}

Input: Two trigonometric polynomials $a$ and $b$.

$$
\begin{aligned}
& (d a, d b) \leftarrow(\operatorname{TD}(a), \operatorname{TD}(b)) \\
& \left(a_{t}, b_{t}\right) \leftarrow\left(\psi_{t}(a) \cdot\left(1+t^{2}\right)^{d a}, \psi_{t}(b) \cdot\left(1+t^{2}\right)^{d b}\right) \\
& g_{t} \leftarrow \operatorname{gcd}\left(a_{t}, b_{t}\right) \\
& \left(\overline{a_{t}}, \overline{b_{t}}\right) \leftarrow\left(a_{t} / g_{t}, b_{t} / g_{t}\right) \\
& l \leftarrow \min \left(d a-\left\lceil\operatorname{deg}_{t}\left(\overline{a_{t}}\right) / 2\right\rceil, d b-\left\lceil\operatorname{deg}_{t}\left(\overline{b_{t}}\right) / 2\right\rceil\right) \\
& \text { if } \operatorname{deg}_{t}\left(g_{t}\right)>2 \cdot l \text { then }
\end{aligned}
$$

$f \leftarrow$ a smallest odd degree factor of $g_{t}$. $g_{t} \leftarrow g_{t} / f, \quad \overline{a_{t}} \leftarrow \overline{a_{t}} \cdot f, \quad \overline{b_{t}} \leftarrow \overline{b_{t}} \cdot f$. $l \leftarrow \min \left(d a-\left\lceil\operatorname{deg}_{t}\left(\overline{a_{t}}\right) / 2\right\rceil, d b-\left\lceil\operatorname{deg}_{t}\left(\overline{b_{t}}\right) / 2\right\rceil\right)$.

$$
g \leftarrow \psi_{t}^{-1}\left(\frac{g_{t}}{\left(1+t^{2}\right)^{l}}\right)
$$

\section{$\operatorname{RETURN}(\mathrm{g})$.}

In the algorithm there is an extra adjustment that has to be made if the degree of $g_{t}$ is too big. Example 5 illustrated this. Note that with condition (iii) the GCD is still nonunique as illustrated by example 4 .
Note, in the algorithm we may need to remove from $p \in \mathbb{Q}[t]$ a factor of least odd degree. This can be done by factoring $p$ over $\mathbb{Q}$ but it makes the trigonometric GCD algorithm relatively expensive. In some applications it will be worthwhile computing any linear factors of $p$ before attempting a full factorization of $p$.

Since we now know how to compute trigonometric GCDs, simplifying a ratio of trigonometric polynomials should be straight forward, namely, compute and divide out by a trigonometric GCD. However, it turns out that we may be able to simplify the ratio further than by dividing out by a trigonometric GCD. Consider the following example.

Example 6: Let $a=5 c^{3}+21 c^{2}+4 c s+23 c+15+12 s$, $b=7 c^{3}-c^{2} s+31 c^{2}+2 c s+37 c+15 s+21$. The images under the t-substitution are:

$$
\psi_{t}(a)=\frac{8\left(t^{2}+2\right)\left(t^{3}+2\right)(t+2)}{\left(1+t^{2}\right)^{3}}
$$

and

$$
\psi_{t}(b)=\frac{8\left(t^{2}+2\right)\left(t^{3}+2\right)(t+3)}{\left(1+t^{2}\right)^{3}} .
$$

Now,

$$
\frac{\psi_{t}(a)}{\psi_{t}(b)}=\frac{t+2}{t+3}=\frac{\frac{t+2}{1+t^{2}}}{\frac{t+3}{1+t^{2}}} .
$$

Converting both the numerator and the denominator back to trigonometric polynomials we get

$$
\frac{a}{b}=\frac{2 c+s+2}{3 c+s+3} \text {. }
$$

So $\frac{a}{b}$ can be simplified to a ratio of two degree 1 trigonometric polynomials. This suggests that a trigonometric polynomial of trigonometric degree 2 must have been cancelled out from both $a$ and $b$. But the only trigonometric GCD of $a$ and $b$ is $c+3$ which has trigonometric degree 1 . There is no common divisor of $a$ and $b$ with trigonometric degree greater than 1 . This implies that $2 c+s+2$ does not divide $a$ and $3 c+s+3$ does not divide $b$.

The previous example illustrates that simplifying rational trigonometric polynomials done via the t-substitution may produce a rational trigonometric polynomial where the trigonometric degrees of the numerator and denominator have dropped more than that of just cancelling the trigonometric GCD. In fact, simplifying this way we are guaranteed that we will not do any worse than if we had cancelled a trigonometric GCD. We prove this in the theorem following the statement of the algorithm. Notice also that we do not need to factor in $\mathbb{Q}[t]$ as we might have to if we attempt to cancel a trigonometric GCD. We now present the algorithm for simplifying rational trigonometric polynomials done via the t-substitution.

\section{Algorithm [trig simplify]}

Input: A rational trigonometric polynomial $\frac{a}{b}$.

$$
\begin{aligned}
& (d a, d b) \leftarrow(\operatorname{TD}(a), \operatorname{TD}(b)) \\
& \left(a_{t}, b_{t}\right) \leftarrow\left(\psi_{t}(a) \cdot\left(1+t^{2}\right)^{d a}, \psi_{t}(b) \cdot\left(1+t^{2}\right)^{d b}\right) \\
& g_{t} \leftarrow \operatorname{gcd}\left(a_{t}, b_{t}\right)
\end{aligned}
$$




$$
\begin{aligned}
& \left(\overline{a_{t}}, \overline{b_{t}}\right) \leftarrow\left(a_{t} / g_{t}, b_{t} / g_{t}\right) \\
& \text { if } d a \leq d b \text { then } \\
& \text { else } \quad \overline{a_{t}} \leftarrow \overline{a_{t}}\left(1+t^{2}\right)^{d b-d a} \\
& \qquad \overline{b_{t}} \leftarrow \overline{b_{t}}\left(1+t^{2}\right)^{d a-d b} \\
& \quad l \leftarrow \max \left(\left\lceil\operatorname{deg}_{t}\left(\overline{a_{t}}\right) / 2\right\rceil,\left\lceil\operatorname{deg}_{t}\left(\overline{b_{t}}\right) / 2\right\rceil\right) \\
& \bar{a} \leftarrow \psi_{t}^{-1}\left(\overline{a_{t}} /\left(1+t^{2}\right)^{l}\right) \\
& \bar{b} \leftarrow \psi_{t}^{-1}\left(\overline{b_{t}} /\left(1+t^{2}\right)^{l}\right) \\
& \operatorname{RETURN}(\bar{a} / \bar{b}) .
\end{aligned}
$$

TheOrem 4. Let $f, g \in \mathbb{Q}[s, c] /\left\langle s^{2}+c^{2}-1\right\rangle$ be non-zero trigonometric polynomials. Let $p / q$ be the output of algorithm trig simplify. Then there does not exist trigonometric polynomials $a, b$ satisfying $a / b=p / q$ and $\mathrm{TD}(a)+\mathrm{TD}(b)<\mathrm{TD}(p)+\mathrm{TD}(q)$.

Proof: Let $d p=\operatorname{TD}(p), d q=\operatorname{TD}(q)$. Let $p_{t}=\psi_{t}(p)(1+$ $\left.t^{2}\right)^{d p}$ and $q_{t}=\psi_{t}(q)\left(1+t^{2}\right)^{d q}$. We claim that the output of algorithm trig simplify satisfies $\operatorname{GCD}\left(p_{t}, q_{t}\right)=1$ and either (i) $\operatorname{deg} p_{t}>2 d p-2$ or (ii) $\operatorname{deg} q_{t}>2 d q-2$, that is $p$ and $q$ are not both divisible by $1+c$. Let $d a=\mathrm{TD}(a), d b=\mathrm{TD}(b)$ and let $a_{t}=\psi_{t}(a)\left(1+t^{2}\right)^{d a}$ and $b_{t}=\psi_{t}(b)\left(1+t^{2}\right)^{d b}$. Now $a / b=p / q$ implies

$$
b_{t} p_{t}\left(1+t^{2}\right)^{d q-d p}=q_{t} a_{t}\left(1+t^{2}\right)^{d b-d a} .
$$

Since $1+t^{2}$ is relatively prime to $a_{t}, b_{t}, p_{t}, q_{t}$ then $d q-d p=$ $d b-d a$, that is,

$$
d q+d a=d b+d p .
$$

Next we note that because $\operatorname{GCD}\left(p_{t}, q_{t}\right)=1$ and $\operatorname{GCD}\left(p_{t}, 1+\right.$ $\left.t^{2}\right)=1$ then $p_{t} \mid a_{t}$. In case (i) we have $\operatorname{deg} p_{t}>2 d p-2$. Since $p_{t} \mid a_{t}$ then we have $2 d a \geq \operatorname{deg} a_{t} \geq \operatorname{deg} p_{t}>2 d p-2$, hence $d a \geq d p$, equivalently $-d a \leq-d p$. Adding this to the above equation yields $d q \leq d b$ hence the theorem follows for this case. A similar argument may be used for case (ii).

\section{OTHER APPROACHES}

One of the approaches tried by Maple 6's simplify command when given a ratio of trigonometric polynomials is the following. Replacing $s$ by $\sqrt{1-c^{2}}$ we may view a ratio of trigonometric polynomials as an algebraic function, that is, an element of the function field $\mathbb{Q}\left(c, \sqrt{1-c^{2}}\right)$. A standard form for algebraic functions is to clear $\sqrt{1-c^{2}}$ from the denominator. Replacing $\sqrt{1-c^{2}}$ back by $s$ yields a result of form $a(c)+b(c) s$ where $a(c)$ and $b(c)$ are in $\mathbb{Q}(c)$. When applied to the simplification problem in the introduction this yields the following result

$$
\frac{\left(c^{2}+c-1\right)}{c\left(c^{4}-3 c^{2}+3\right)} s-\frac{(1+c)\left(c^{2}-c-1\right)}{c\left(c^{4}-3 c^{2}+3\right)} .
$$

We observe that the trigonometric degree of the numerator is 3 and that of the denominator is 5 which is greater than that of the input, thus this is not the simplest form even though it is a canonical form.

The approach taken by REDUCE 7's trigsimp command [1] when given an input involving $\sin x$ and $\cos x$ is to apply rewriting techniques similar to those used by the simplify and combine commands in Maple 6. The numerator and denominator of a ratio of trigonometric polynomials is put in a canonical form but there is no guarantee that a common factor of the numerator and denominator will be detected and cancelled out. REDUCE 7 succeeds on the example in the introduction but fails on example 5 .

The approach taken by REDUCE 7's trigfactorize and triggcd commands [1] is to apply the method of Roach [3]. We give some details of this method here. Roach's method first converts inputs to complex exponentials. Let $i=\sqrt{-1}$ and $e$ denote $e^{i x}$. Let $f \in \mathbb{Q}[s, c] /\left\langle s^{2}+c^{2}-1\right\rangle$ and $g \in$ $\mathbb{Q}(i)\left[e, e^{-1}\right]$ be the image of $f$ under the complex exponential map.

Definition 9. $g \in \mathbb{Q}(i)\left[e, e^{-1}\right]$ is co-ordinated if $g(e)$ is an even or odd function of $e$.

Example 7: $s=\frac{i}{2} e^{-1}-\frac{i}{2} e$ and $s c=\frac{i}{4} e^{-2}-\frac{i}{4} e^{2}$ are coordinated but $s+1=\frac{i}{2} e^{-1}+1-\frac{i}{2} e$ and $s^{2}+s=-\frac{1}{4} e^{-2}+$ $\frac{i}{2} e+\frac{1}{2}-\frac{i}{2} e-\frac{1}{4} e^{2}$ are not co-ordinated.

To factor a trigonometric polynomial Roach requires that it be co-ordinated. We remark that most trigonometric polynomials are not co-ordinated. Roach multiplies through by the least power of $e$ so that the input is now in $\mathbb{Q}(i)[e]$. Notice that the input will now be an even polynomial of $e$ so the substitution $e \rightarrow \sqrt{e}$ can be made. Roach then factors the resulting polynomial in $\mathbb{Q}(i)[e]$ and reverses this process to obtain a factorization in $\mathbb{Q}[s, c] /\left\langle s^{2}+c^{2}-1\right\rangle$. The procedure for computing a GCD of co-ordinated polynomials in $\mathbb{Q}[s, c] /\left\langle s^{2}+c^{2}-1\right\rangle$ is analogous. The key observation made by Roach is that if a trigonometric polynomial is coordinated and irreducible then when this procedure is applied to express it in $\mathbb{Q}(i)[e]$, it will be irreducible. Because $\mathbb{Q}(i)[e]$ is a UFD, these procedures will return unique results for any given input, however, this does not mean that the factorization and GCD is unique.

Example 8: To factor $s c=\frac{i}{4} e^{-2}-\frac{i}{4} e^{2}$ Roach's algorithm factors the polynomial $\frac{i}{4}-\frac{i}{4} e^{2}=\frac{i}{4}(e+1)(e-1)$ from which one recovers the factorization $s \times c$, not the two other factorizatons shown in example 2 .

To deal with the non co-ordinated case, Roach suggests that one make the substitution $x \rightarrow 2 x$ to the input polynomials. This makes them co-ordinated. But, reversing the substitution results in outputs involving $\sin \frac{x}{2}$ and $\cos \frac{x}{2}$ of double the degree.

Example 9: To factor $\sin (x)+1$ and $\sin (x)^{2}+\sin x$ one factors the polynomials $-i e^{2}+2 e+i=-i(e+i)^{2}$ and $-\frac{1}{4}\left(e^{4}+2 i e^{3}-2 e^{2}-2 i e+1\right)=-\frac{1}{4}(e-1)(e+1)(e+i)^{2}$ respectively. This yields the factorizations $\left(\sin \frac{x}{2}+\cos \frac{x}{2}\right)^{2}$ and $2 \sin \frac{x}{2} \cos \frac{x}{2}\left(\sin \frac{x}{2}+\cos \frac{x}{2}\right)^{2}$ respectively.

The REDUCE 7 trigfactorize command, on input of trigonometric polynomials which are not co-ordinated returns the input polynomials unfactored. Similarly, the triggcd command returns 1 for the GCD. The user may specify $\frac{x}{2}$ as an option to either command to effect the $x \rightarrow 2 x$ substitution just described. 


\section{CONCLUSIONS AND FINAL REMARKS}

The main result of our paper is a way to simplify ratios of trigonometric polynomials. We have also shown how to divide, factor and compute GCDs of trigonometric polynomials. Though we have not given the algorithmic details for dividing and factoring trigonometric polynomials in the paper, we have implemented all of these operations in Maple. Our Maple code is available from the authors or from our web site ww. cecm.sfu.ca/CAG/products.html.

The theory for factorization of trigonometric polynomials which we developed over $\mathbb{Q}$ holds for any constant field $k$ provided $i=\sqrt{-1} \notin k$. If $i \in k$ then $s+i c$ and $s-i c$ are units as $(s+i c)(s-i c)=1$ and consequently the trigonometric degree sum formula that we gave no longer holds. We have not modified the theory for this case but we note that it will be simpler because $\mathbb{Q}(i)[s, c] /\left\langle s^{2}+c^{2}-1\right\rangle$ is a UFD, thus GCDs are unique up to multiplication by units. Consequently simplification can be accomplished by cancelling a GCD.

\section{Acknowledgement}

We acknowledge helpful discussions with our colleagues, in particular Hans Bauck, Mark van Hoeij, and Petr Lisonek. We also thank Wolfram Koepf for providing us with information about REDUCE's trigonometric polynomial commands and the referees for their comments.

\section{REFERENCES}

[1] Koepf, W., Bernig A., Melenk H. (1999) TRIGSIMP: A REDUCE Package for the Simplification and Factorization of Trigonometric and Hyperbolic Expressions. REDUCE 7 documentation.

[2] Hungerford, T.W. (1980) Algebra, second edition, Graduate Texts in Mathematics, Springer-Verlag.

[3] Roach, K. (1992), Trigonometric Factorization and Integration, Unpublished manuscript. Presented at the Maple Retreat, June, 1992.

[4] Trotter, H.F. (1989), An Overlooked Example of Nonunique Factorization, American Mathematical Monthly, 95(4), 339-342. 